\title{
Filter integrals for orthogonal polynomials
}

\author{
T. Amdeberhan, Adriana Duncan, Victor H. Moll and Vaishavi Sharma
}

Dedicated to the memory of Srinivasa Ramanujan

\begin{abstract}
Motivated by an expression by Persson and Strang on an integral involving Legendre polynomials, stating that the square of $P_{2 n+1}(x) / x$ integrated over $[-1,1]$ is always 2 , we present analog results for Hermite, Chebyshev, Laguerre and Gegenbauer polynomials as well as the original Legendre polynomial with even index.
\end{abstract}

Keywords. Integrals, Legendre polynomials, Hermite polynomials, Chebyshev polynomials, Laguerre polynomials, Gegenbauer polynomials.

2010 Mathematics Subject Classification. Primary 33C45, Secondary 33E20.

\section{Introduction}

Persson and Strang [PeSt03] presented a family of filters, symmetric of even degree $n$, obtained by sampling a multiple of the polynomial function $x^{-1} P_{n+1}(x)$, where $P_{n}(x)$ is the Legendre polynomial. In their analysis of the error due to noise, they proved Theorem 1.1 below. The authors commented that this result was not expected to them.

Theorem 1.1. For all $n \in \mathbb{N}$,

$$
\int_{-1}^{1}\left(\frac{P_{2 n+1}(x)}{x}\right)^{2} d x=2
$$

The goal of this note is to present extensions of Theorem 1.1 to other families of orthogonal polynomials $\left\{A_{n}(x)\right\}$, with weight $w_{A}(x)$. The integrals evaluated here have the form

$$
I_{n}(a, b ; w)=\int_{a}^{b}\left(\frac{A_{n}(x)-A_{n}(0)}{x}\right)^{2} w_{A}(x) d x,
$$

where the term $A_{n}(0)$ is introduced to guarantee convergence of the integral.

The rest of this introduction states our results. The polynomials considered here include Legendre, Hermite, Chebyshev, Laguerre and Gegenbauer. Some basic properties of each of these polynomials are in the introduction to the corresponding sections.

Section 2 contains the evaluation complementary to (1.1) for even indexed Legendre polynomials. The statement is given in terms of

$$
\beta(n)= \begin{cases}0 & \text { if } n \text { is odd } \\
2^{-n}\left(\begin{array}{c}
n \\
n / 2
\end{array}\right) & \text { if } n \text { is even. }\end{cases}
$$

Theorem 1.2. For $n \in \mathbb{N}$ and $P_{n}$ the Legendre polynomial,

$$
\int_{-1}^{1}\left(\frac{P_{n}(x)-P_{n}(0)}{x}\right)^{2} d x=2\left[1-\beta^{2}(n)\right]
$$

We thank episciences.org for providing open access hosting of the electronic journal Hardy-Ramanujan Journal 
Section 3 contains the analog to Theorem 1.2 for Hermite polynomials $H_{n}(x)$. These form a family of orthogonal polynomials on $\mathbb{R}$ with respect to the weight $w_{H}(x)=e^{-x^{2}}$.

Theorem 1.3. For $n \in \mathbb{N}$ and $H_{n}$ the Hermite polynomial, then

$$
\int_{-\infty}^{\infty}\left(\frac{H_{n}(x)-H_{n}(0)}{x}\right)^{2} e^{-x^{2}} d x=\sqrt{\pi} n ! 2^{n+1}[1-\beta(n)] .
$$

Section 4 analyzes the corresponding integral for Chebyshev polynomials of both kinds $\left\{T_{n}\right\}$ and $\left\{U_{n}\right\}$. These are defined by the relations

$$
\cos (n x)=T_{n}(\cos x) \quad \text { and } \quad \frac{\sin ((n+1) x)}{\sin x}=U_{n}(\cos x) .
$$

The family $\left\{T_{n}\right\}$ is orthogonal with respect to the weight $\left(1-x^{2}\right)^{-1 / 2}$ and $\left\{U_{n}\right\}$ with respect to $\left(1-x^{2}\right)^{1 / 2}$ on the interval $[-1,1]$.

Theorem 1.4. For $n \in \mathbb{N}$,

$$
\begin{aligned}
\int_{-1}^{1}\left[\frac{T_{n}(x)-T_{n}(0)}{x}\right]^{2} \frac{d x}{\sqrt{1-x^{2}}} & =\pi n, \\
\int_{-1}^{1}\left[\frac{U_{n}(x)-U_{n}(0)}{x}\right]^{2} \sqrt{1-x^{2}} d x & = \begin{cases}\pi n & \text { for } n \text { even } \\
\pi(n+1) & \text { if } n \text { is odd } .\end{cases}
\end{aligned}
$$

Section 5 describes the evaluation of the corresponding integrals for the family of a special case of the Laguerre polynomials, corresponding to the parameter $\alpha$ being a nonnegative integer. This special case forms an orthogonal family of polynomials on $[0, \infty)$ with the weight function $w_{L}(x)=x^{\alpha} e^{-x}$.

Theorem 1.5. For $n \in \mathbb{N}$, let $L_{n}^{\alpha}(x)$ be the Laguerre polynomial with $\alpha$ restricted to be a nonnegative integer. Then, for $\alpha=0$,

$$
\int_{0}^{\infty}\left[\frac{L_{n}^{0}(x)-L_{n}^{0}(0)}{x}\right]^{2} e^{-x} d x=2 n-H_{n}
$$

where $H_{n}$ is the harmonic number. For $\alpha=1$,

$$
\int_{0}^{\infty}\left[\frac{L_{n}^{1}(x)-L_{n}^{1}(0)}{x}\right]^{2} x e^{-x} d x=-\frac{1}{2}(3 n+2)(n+1)+(n+1)^{2} H_{n+1}
$$

and for $\alpha \geq 2$ integer,

$$
\int_{0}^{\infty}\left[\frac{L_{n}^{\alpha}(x)-L_{n}^{\alpha}(0)}{x}\right]^{2} x^{\alpha} e^{-x} d x=(\alpha-2) !\left(\begin{array}{c}
\alpha+n \\
\alpha
\end{array}\right)\left[\left(\begin{array}{c}
\alpha+n \\
n
\end{array}\right) \alpha-\frac{2 n \alpha+\alpha+1}{\alpha+1}\right] .
$$

Finally, Section 6 discusses the Gegenbauer polynomials $C_{n}^{(a)}(x)$. These are defined by the recurrence

$$
C_{n}^{(a)}(x)=\frac{1}{n}\left[2 x(n+a-1) C_{n-1}^{(a)}(x)-(n+2 a-2) C_{n-2}^{(a)}(x)\right],
$$

with initial conditions $C_{0}^{(a)}(x)=1$ and $C_{1}^{(a)}(x)=2 a x$. The Gegenbauer polynomials are orthogonal with respect to the weight $\left(1-x^{2}\right)^{a-1 / 2}$ and are normalized by

$$
\int_{-1}^{1}\left[C_{n}^{a}(x)\right]^{2}\left(1-x^{2}\right)^{a-1 / 2} d x=\frac{\pi \Gamma(n+2 a)}{2^{2 a-1} n !(n+a) \Gamma^{2}(a)} .
$$

The corresponding result is: 
Theorem 1.6. For $n \in \mathbb{N}$, let $C_{n}^{a}(x)$ be the Gegenbauer polynomial. Then

$$
\gamma_{n}(a)=\int_{-1}^{1}\left[\frac{C_{n}^{a}(x)-C_{n}^{a}(0)}{x}\right]^{2}\left(1-x^{2}\right)^{a-1 / 2} d x
$$

where

$$
\gamma_{2 n+1}(a)=\frac{\pi \Gamma(2 a+2 n+1)}{2^{2 a-2}(2 n+1) ! \Gamma^{2}(a)}
$$

for an odd index and, in the case of even index,

$$
\gamma_{2 n}(a)=\frac{\pi \Gamma(2 a) \Gamma(a+n)}{2^{2 a-3} \Gamma(2 n+1) \Gamma^{3}(a)} X_{n}(a),
$$

where $X_{n}(a)$ is the polynomial

$$
X_{n}(a)=2^{2 n-1}\left(a+\frac{1}{2}\right)_{n}-\left(\begin{array}{c}
2 n-1 \\
n-1
\end{array}\right)(a)_{n}
$$

The last section contains some conjectures on the polynomial $X_{n}(a)$.

\section{The extension of Legendre integrals to even indices}

The proof of Theorem 1.1 in [PeSt03] starts with the classical recurrence

$$
(n+1) P_{n+1}(x)-(2 n+1) x P_{n}(x)+n P_{n-1}(x)=0
$$

satisfied by the Legendre polynomials. This appears as entry 7.221.1 in [GrRy15]. Divide (2.16) by $x$ and write $n=2 m$ to obtain

$$
(2 m+1) \frac{P_{2 m+1}(x)}{x}-(4 m+1) P_{2 m}(x)+2 m \frac{P_{2 m-1}(x)}{x}=0 .
$$

Since $P_{k}(0)=0$ for $k$ odd, this is a polynomial relation.

Define

$$
c_{m}=\int_{-1}^{1}\left(\frac{P_{2 m-1}(x)}{x}\right)^{2} d x .
$$

Square (2.17) and integrate over $[-1,1]$ to obtain

$$
(2 m+1)^{2} c_{m+1}=(4 m+1)^{2} \int_{-1}^{1} P_{2 m}^{2}(x) d x-4 m(4 m+1) \int_{-1}^{1} P_{2 m}(x) \frac{P_{2 m-1}(x)}{x} d x+4 m^{2} c_{m} .
$$

The integral

$$
\int_{-1}^{1} P_{2 m}^{2}(x) d x=\frac{2}{4 m+1}
$$

is well-known (see entry 7.221.1 in [GrRy15]). The other integral is zero since $P_{2 m-1}(x) / x$ is a polynomial of degree $2 m-2$, so it is orthogonal to $P_{2 m}(x)$. It follows that

$$
(2 m+1)^{2} c_{m+1}=2(4 m+1)+4 m^{2} c_{m-1} .
$$

The initial value $c_{1}=2$ shows that $c_{2 m+1}=2$ for all $m \in \mathbb{N}$.

The goal of this section is to present the result given in Theorem 1.1 for the case of an even index $n$. The polynomial $P_{n}$ does not vanish at $x=0$, so it is necessary to modify the integrand slightly. 
Theorem 2.1. For all $m \in \mathbb{N}$,

$$
\int_{-1}^{1}\left(\frac{P_{2 m}(x)-P_{2 m}(0)}{x}\right)^{2} d x=2\left[1-\frac{1}{2^{4 m}}\left(\begin{array}{c}
2 m \\
m
\end{array}\right)^{2}\right] .
$$

The proof of this assertion begins with (2.16) for $n=2 m+1$, in the form,

$$
(2 m+2)\left[P_{2 m+2}(x)-P_{2 m+2}(0)\right]-(4 m+3) x P_{2 m+1}(x)+(2 m+1)\left[P_{2 m}(x)-P_{2 m}(0)\right]=0 .
$$

The contribution of the polynomials at $x=0$ vanishes since

$$
-(2 m+2) P_{2 m+2}(0)-(2 m+1) P_{2 m}(0)=0
$$

in view of

$$
P_{2 m}(0)=\frac{(-1)^{m}\left(\begin{array}{c}
2 m \\
m
\end{array}\right)}{2^{2 m}}
$$

Then (2.22) may be written as

$$
2(m+1)\left[\frac{P_{2 m+2}(x)-P_{2 m+2}(0)}{x}\right]=(4 m+3) P_{2 m+1}(x)-(2 m+1)\left[\frac{P_{2 m}(x)-P_{2 m}(0)}{x}\right] .
$$

Squaring this relation produces

$$
\begin{aligned}
4(m+1)^{2}\left[\frac{P_{2 m+2}(x)-P_{2 m+2}(0)}{x}\right]^{2}= & (4 m+3)^{2} P_{2 m+1}^{2}(x) \\
& -2(4 m+3)(2 m+1) P_{2 m+1}(x) \times\left[\frac{P_{2 m}(x)-P_{2 m}(0)}{x}\right] \\
& +(2 m+1)^{2}\left[\frac{P_{2 m}(x)-P_{2 m}(0)}{x}\right]^{2} .
\end{aligned}
$$

Denote

$$
w_{m}=\int_{-1}^{1}\left[\frac{P_{2 m}(x)-P_{2 m}(0)}{x}\right]^{2} d x
$$

integrate (2.26) from $x=-1$ to +1 and use (2.19)

$$
\int_{-1}^{1} P_{2 m+1}^{2}(x) d x=\frac{2}{4 m+3} .
$$

Also apply the relation

$$
\int_{-1}^{1} P_{2 m+1}(x) \times\left[\frac{P_{2 m}(x)-P_{2 m}(0}{x}\right] d x=0
$$

coming from the orthogonality of $P_{2 m+1}(x)$ and $\left(P_{2 m}(x)-P_{2 m}(0)\right) / x$, the latter being a polynomial of degree $2 m-1$. This proves the next statement.

Lemma 2.2. The integrals $w_{m}$ defined in (2.27) satisfy the recurrence

$$
4(m+1)^{2} w_{m+1}=2(4 m+3)+(2 m+1)^{2} w_{m},
$$

with initial condition $w_{0}=0$. 
The expression for $w_{m}$ in Theorem 2.1 follows directly from Lemma 2.2. Indeed, the solution $w_{m}$ of (2.30) has the form $w_{m}=w_{m}^{(h)}+w_{m}^{(p)}$, where $w_{m}^{(p)}$ is a particular solution and $w_{m}^{(h)}$ is the general solution of the homogeneous equation

$$
(2 m+2)^{2} w_{m+1}^{(h)}=(2 m+1)^{2} w_{m}^{(h)} .
$$

Since the coefficients of $w_{m+1}$ and $w_{m}$ in (2.30) have the same degree, one tries as a particular solution a constant $w_{m}^{(p)}=C$. Replacing in (2.30) yields $C=2$. The general solution of the homogeneous equation (2.31) is obtained by iterating the recurrence to obtain

$$
w_{m}^{(h)}=\left[\frac{[(2 m-1)(2 m-3) \cdots 3 \cdot 1}{(2 m)(2 m-2) \cdots 4 \cdot 2}\right]^{2}=2^{-4 m}\left(\begin{array}{c}
2 m \\
m
\end{array}\right)^{2}
$$

up to a scalar. Therefore, the solution of (2.30) has the form

$$
w_{m}=2+\alpha 2^{-4 m}\left(\begin{array}{c}
2 m \\
m
\end{array}\right)^{2} .
$$

The initial condition $w_{0}=0$ gives $\alpha=-2$ proving Theorem 2.1.

\section{The Hermite case}

The Hermite polynomials $\left\{H_{n}(x)\right\}$ form a family of orthogonal polynomials on $\mathbb{R}$ with respect to a gaussian weight $e^{-x^{2}}$ with the normalization condition (appearing as entry 8.959(1).2 in [GrRy15])

$$
\int_{-\infty}^{\infty} H_{n}(x) H_{m}(x) e^{-x^{2}} d x=\sqrt{\pi} 2^{n} n ! \delta_{n m}
$$

where $\delta_{n m}$ is the Kronecker delta. This family is the so-called physicists Hermite, a second family with $\frac{1}{2} x^{2}$ instead of $x^{2}$ in the exponent of the weight also appears in the literature. The latter are the probabilists Hermite, so one should be careful in checking identities.

As part of a general procedure in extending Theorem 1.1, E. Diekema and T. Koorwinder [DiKo12] established the next statement.

Theorem 3.1. For all $n \in \mathbb{N}$, define

$$
I_{2 n+1}=\int_{-\infty}^{\infty}\left(\frac{H_{2 n+1}(x)}{x}\right)^{2} e^{-x^{2}} d x .
$$

Then

$$
I_{2 n+1}=\sqrt{\pi} 2^{2 n+2}(2 n+1) !
$$

The goal of this section is to determine the analog of (3.35) for even indices. Introduce the notation

$$
I_{2 n}=\int_{-\infty}^{\infty}\left(\frac{H_{2 n}(x)-H_{2 n}(0)}{x}\right)^{2} e^{-x^{2}} d x
$$

complementary to (3.35). Symbolic evaluation using Mathematica shows that

$$
O_{n}=\frac{I_{2 n}}{2^{2 n+1} \sqrt{\pi}}
$$

is an odd integer sequence, starting with 1, 15, 495, 29295, 2735775. A search in OEIS (the Online Encyclopedia of Integer Sequences) produced entry A151816. This is how the statement of the next theorem was obtained. 
Theorem 3.2. For $n \in \mathbb{N}$ the integral $I_{2 n}$ defined in (3.37) is computed by

$$
I_{2 n}=2 \sqrt{\pi}(2 n) !\left[2^{2 n}-\left(\begin{array}{c}
2 n \\
n
\end{array}\right)\right] .
$$

Note 3.3. The results stated in Theorems 3.1 and 3.2 can be combined as

$$
I_{k}=2 \sqrt{\pi} k !\left[2^{k}-\left\{\begin{array}{ll}
0 & \text { if } k \text { is odd } \\
\left(\begin{array}{c}
k \\
k / 2
\end{array}\right) & \text { if } k \text { is even }
\end{array}\right\} .\right.
$$

The proof of Theorem 3.2 begins with the recurrence

$$
H_{n+1}(x)=2 x H_{n}(x)-2 n H_{n-1}(x),
$$

listed as entry 8.952.2 in [GrRy15]. Now let $n=2 m+1$ to obtain

$$
\frac{H_{2 m+2}(x)-H_{2 m+2}(0)}{x}=2 x\left[\frac{H_{2 m+1}(x)-H_{2 m+1}(0)}{x}\right]-2(2 m+1)\left[\frac{H_{2 m}(x)-H_{2 m}(0)}{x}\right]
$$

where extra terms appearing in the previous identity, namely those with values of $H_{n}(x)$ at $x=0$, cancel each other using

$$
H_{n}(0)= \begin{cases}0 & \text { if } n \text { is odd } \\ (-1)^{n / 2} \frac{n !}{(n / 2) !} & \text { if } n \text { is even. }\end{cases}
$$

Since $H_{2 m+1}(0)=0,(3.42)$ becomes

$$
\frac{H_{2 m+2}(x)-H_{2 m+2}(0)}{x}=2 H_{2 m+1}(x)-2(2 m+1)\left[\frac{H_{2 m}(x)-H_{2 m}(0)}{x}\right] .
$$

Squaring gives

$$
\begin{aligned}
{\left[\frac{H_{2 m+2}(x)-H_{2 m+2}(0)}{x}\right]^{2}=} & 4 H_{2 m+1}^{2}(x)-8(2 m+1) H_{2 m+1}(x)\left[\frac{H_{2 m}(x)-H_{2 m}(0)}{x}\right] \\
& +4(2 m+1)^{2}\left[\frac{H_{2 m}(x)-H_{2 m}(0)}{x}\right]^{2}
\end{aligned}
$$

Now multiply by the weight $e^{-x^{2}}$ and integrate over $(-\infty, \infty)$. The integral coming from the second term vanishes since the bracketed expression is a polynomial of degree $2 m-1$, so it is orthogonal to $H_{2 m+1}(x)$. From the normalization (3.34) one obtains the recurrence

$$
I_{2 m+2}=\sqrt{\pi}(2 m+1) ! 2^{2 m+3}+4(2 m+1)^{2} I_{2 m} .
$$

This is complemented by the initial data $I_{0}=0$. Motivated by data generated from (3.45), introduce the auxiliary unknown

$$
J_{m}=\frac{I_{2 m}}{\sqrt{\pi} 2^{2 m+1}(2 m) !}
$$

and reduce (3.45) to

$$
(2 m+2) J_{m+1}=(2 m+1) J_{m}+1 \quad \text { with } J_{0}=0 .
$$

Lemma 3.4. The solution to (3.47) is given by

$$
J_{m}=1-2^{-2 m}\left(\begin{array}{c}
2 m \\
m
\end{array}\right) .
$$


Proof. A particular solution of (3.48) is $J_{m}=1$. The homogeneous part

$$
(2 m+2) J_{m+1}=(2 m+1) J_{m}
$$

has a general solution

$$
J_{m}^{(h)}=2^{-2 m}\left(\begin{array}{c}
2 m \\
m
\end{array}\right)
$$

so that $J_{m}=1+\alpha J_{m}^{(h)}$ for some constant $\alpha$. The value $J_{0}=0$ gives $\alpha=-1$. This completes the proof.

The expression for $J_{m}$ now gives the evaluation for $I_{2 m}$ in Theorem 3.2.

\section{The Chebyshev case}

The Chebyshev polynomials come in two flavors. The first kind $T_{n}(x)$, defined by

$$
T_{n}(\cos x)=\cos (n x)
$$

form an orthogonal family on $[-1,1]$ with respect to the weight $\left(1-x^{2}\right)^{-1 / 2}$ and satisfy

$$
\int_{-1}^{1} \frac{T_{n}^{2}(x) d x}{\sqrt{1-x^{2}}}= \begin{cases}\pi & \text { if } n=0 \\ \frac{\pi}{2} & \text { if } n \neq 0 .\end{cases}
$$

These polynomials satisfy the recurrence $T_{n+1}(x)=2 x T_{n}(x)-T_{n-1}(x)$ (for $n \geq 1$ ) with initial conditions $T_{0}(x)=1$ and $T_{1}(x)=x$.

The Chebyshev polynomials of the second kind $U_{n}(x)$, defined by

$$
U_{n}(\cos x)=\frac{\sin ((n+1) x)}{\sin x},
$$

are orthogonal on $[-1,1]$ with respect to the weight $\left(1-x^{2}\right)^{1 / 2}$ normalized by

$$
\int_{-1}^{1} U_{n}^{2}(x) \sqrt{1-x^{2}} d x=\frac{\pi}{2}
$$

These polynomials satisfy the recurrence $U_{n+1}(x)=2 x U_{n}(x)-U_{n-1}(x)$ (for $n \geq 1$ ) with initial conditions $U_{0}(x)=1$ and $U_{1}(x)=2 x$. This is the same recurrence as the one satisfied by $T_{n}(x)$.

The relevant integrals considered in the current work, in the case of Chebyshev polynomials, are stated next.

Theorem 4.1. For $n \in \mathbb{N}$,

$$
\begin{aligned}
I_{n} & \equiv \int_{-1}^{1}\left[\frac{T_{n}(x)-T_{n}(0)}{x}\right]^{2} \frac{d x}{\sqrt{1-x^{2}}}=\pi n, \\
J_{n} & \equiv \int_{-1}^{1}\left[\frac{U_{n}(x)-U_{n}(0)}{x}\right]^{2} \sqrt{1-x^{2}} d x= \begin{cases}\pi n & \text { if } n \equiv 0 \bmod 2 \\
\pi(n+1) & \text { if } n \equiv 1 \bmod 2 .\end{cases}
\end{aligned}
$$

Proof. The proof proceeds as before. Square the recurrence for $T_{n}$ to obtain

$$
\begin{aligned}
{\left[\frac{T_{n+1}(x)-T_{n+1}(0)}{x}\right]^{2}=} & 4 T_{n}^{2}(x)-4 T_{n}(x) \cdot\left[\frac{T_{n-1}(x)-T_{n-1}(0)}{x}\right] \\
& +\left[\frac{T_{n-1}(x)-T_{n-1}(0)}{x}\right]^{2} .
\end{aligned}
$$


Now divide by $\sqrt{1-x^{2}}$ and integrate over $[-1,1]$. The integral coming from the second line above vanishes since $\left(T_{n-1}(x)-T_{n-1}(0)\right) / x$ is a polynomial of degree $n-2$ and thus orthogonal to $T_{n}(x)$. With the notation

$$
a_{n}=\int_{-1}^{1}\left[\frac{T_{n}(x)-T_{n}(0)}{x}\right]^{2} \frac{d x}{\sqrt{1-x^{2}}}
$$

and the normalization for $T_{n}(x)$, the recurrence (4.55) yields

$$
a_{n+1}=2 \pi+a_{n-1} \quad \text { for } n>0 .
$$

The initial conditions $a_{1}=\pi$ and $a_{2}=2 \pi$ lead to the result. The proof for $U_{n}(x)$ is similar.

\section{The Laguerre case}

The Laguerre polynomials $L_{n}^{\alpha}(x)$ defined by

$$
L_{n}^{\alpha}(x)=\frac{x^{-\alpha} e^{x}}{n !}\left(\frac{d}{d x}\right)^{n}\left[x^{n+\alpha} e^{-x}\right], \quad n=0,1,2, \ldots \text { and } \alpha>-1
$$

is a one-parameter family of classical orthogonal polynomials. Their explicit formula is given by

$$
L_{n}^{\alpha}(x)=\sum_{k=0}^{n} \frac{\Gamma(\alpha+n+1)}{\Gamma(\alpha+k+1)} \frac{(-x)^{k}}{k !(n-k) !} .
$$

The goal of this section is to present the evaluation of the integral

$$
W_{n}(\alpha)=\int_{0}^{\infty}\left[\frac{L_{n}^{\alpha}(x)-L_{n}^{\alpha}(0)}{x}\right]^{2} e^{-x} x^{\alpha} d x
$$

with particular emphasis in the case when $\alpha$ is a nonnegative integer.

A direct computation using (5.59) yields the expression

$$
W_{n}(\alpha)=(\alpha)_{n+1}^{2}\left[\sum_{j=1}^{n} \sum_{k=1}^{n}(-1)^{j+k} \frac{(\alpha)_{k+j-1}}{(\alpha)_{k+1}(\alpha)_{j+1}}\right] \Gamma(\alpha),
$$

where $(\alpha)_{n}=\alpha(\alpha+1) \cdots(\alpha+n-1)$ is the Pochhammer symbol. It is clear from here that the integral $W_{n}(\alpha)$ is a rational function of $\alpha$ times $\Gamma(\alpha)$.

The details given below makes these expressions explicit in the case $\alpha$ is a nonnegative integer. In this situation, the double sum above can be expressed in terms of more elementary objects. The discussion is divided into three cases $\alpha=0, \alpha \geq 2$ and $\alpha=1$. The placing of $\alpha=1$ is simply because an integral evaluated in the case $\alpha \geq 2$ is used in this last case, so it avoid repetitions.

\section{A. The special case $\alpha=0$.}

The Laguerre polynomial, writing $L_{n}(x)=L_{n}^{0}(x)$, is

$$
L_{n}(x)=\sum_{k=0}^{n} \frac{(-1)^{k}}{k !}\left(\begin{array}{l}
n \\
k
\end{array}\right) x^{k}
$$

as this appears in entry 8.970.1 in [GrRy15].

The goal of this section is dictated by the next theorem. 
Theorem 5.1. For $n \in \mathbb{N}$, let $L_{n}(x)$ be the Laguerre polynomial of special parameter $\alpha=0$. Then

$$
\int_{0}^{\infty}\left[\frac{L_{n}(x)-L_{n}(0)}{x}\right]^{2} e^{-x} d x=2 n-H_{n}
$$

where $H_{n}=1+\frac{1}{2}+\cdots+\frac{1}{n}$ is the harmonic number.

Proof. Square (5.62) to produce

$$
\left[\frac{L_{n}(x)-L_{n}(0)}{x}\right]^{2}=\sum_{k=1}^{n} \sum_{j=1}^{n}\left(\begin{array}{l}
n \\
k
\end{array}\right)\left(\begin{array}{l}
n \\
j
\end{array}\right) \frac{(-1)^{k+j}}{k ! j !} x^{k+j-2} .
$$

Now integrate with respect to the weight $e^{-x}$ and with the notation

$$
B_{n, k}=\sum_{j=1}^{n} \frac{(-1)^{j}}{j}\left(\begin{array}{c}
n \\
j
\end{array}\right)\left(\begin{array}{c}
k+j-2 \\
k-1
\end{array}\right)
$$

write

$$
\int_{0}^{\infty}\left[\frac{L_{n}(x)-L_{n}(0)}{x}\right]^{2} e^{-x} d x=\sum_{k=1}^{n} \frac{(-1)^{k}}{k}\left(\begin{array}{l}
n \\
k
\end{array}\right) B_{n, k} .
$$

The next step is to evaluate $B_{n, k}$.

Lemma 5.2. For $n \in \mathbb{N}$ and $H_{n}$ the harmonic number,

$$
\sum_{k=2}^{n} \frac{(-1)^{k}}{k-1}\left(\begin{array}{l}
n \\
k
\end{array}\right)=n\left(H_{n}-1\right) .
$$

Proof. Let $\alpha_{n}$ be the left-hand side of (5.67). Then

$$
\begin{aligned}
\alpha_{n}-\alpha_{n-1} & =\sum_{k=2}^{n} \frac{(-1)^{k}}{k-1}\left(\begin{array}{l}
n \\
k
\end{array}\right)-\sum_{k=2}^{n-1} \frac{(-1)^{k}}{k-1}\left(\begin{array}{c}
n-1 \\
k
\end{array}\right) \\
& =\frac{(-1)^{n}}{n-1}+\sum_{k=2}^{n-1} \frac{(-1)^{k}}{k-1}\left[\left(\begin{array}{l}
n \\
k
\end{array}\right)-\left(\begin{array}{c}
n-1 \\
k
\end{array}\right)\right] \\
& =\sum_{\ell=1}^{n-1} \frac{(-1)^{\ell+1}}{\ell}\left(\begin{array}{c}
n-1 \\
\ell
\end{array}\right) .
\end{aligned}
$$

Denote this last sum by $W$ and observe that

$$
W=\int_{0}^{1} \frac{1-(1-s)^{n-1}}{s} d s .
$$

The change of variables $t=1-s$ and expanding the new integrand gives $W=H_{n-1}$. Thus, $\alpha_{n}-\alpha_{n-1}=H_{n-1}$. A direct computation shows that the right-hand side of (5.67) satisfies the same difference equation and since the initial values match, the identity (5.67) holds for all $n \in \mathbb{N}$.

Lemma 5.3. For $n \in \mathbb{N}$ and $H_{n}$ the harmonic number,

$$
\sum_{k=2}^{n} \frac{(-1)^{k}}{k}\left(\begin{array}{l}
n \\
k
\end{array}\right)=n-H_{n} .
$$


Proof. Let $b_{n}$ be the left-hand side of (5.70). Then

$$
\begin{aligned}
b_{n}-b_{n-1} & =\sum_{k=2}^{n} \frac{(-1)^{k}}{k}\left(\begin{array}{l}
n \\
k
\end{array}\right)-\sum_{k=2}^{n-1} \frac{(-1)^{k}}{k}\left(\begin{array}{c}
n-1 \\
k
\end{array}\right) \\
& =\frac{(-1)^{n}}{n}+\sum_{k=2}^{n-1} \frac{(-1)^{k}}{k}\left[\left(\begin{array}{l}
n \\
k
\end{array}\right)-\left(\begin{array}{c}
n-1 \\
k
\end{array}\right)\right] \\
& =\sum_{k=2}^{n} \frac{(-1)^{k}}{k}\left(\begin{array}{l}
n-1 \\
k-1
\end{array}\right) .
\end{aligned}
$$

Now use $\frac{1}{k}\left(\begin{array}{l}n-1 \\ k-1\end{array}\right)=\frac{1}{n}\left(\begin{array}{l}n \\ k\end{array}\right)$ to obtain $b_{n}-b_{n-1}=\frac{1}{n} \sum_{k=2}^{n}(-1)^{k}\left(\begin{array}{l}n \\ k\end{array}\right)=1-\frac{1}{n}$ using the fact that the alternating sum of binomial coefficients vanish. A direct computation shows that $n-H_{n}$ satisfies the same difference equation and has the same initial condition as $b_{n}$. This proves the identity.

Lemma 5.4. For $n \in \mathbb{N}$ and with the notation in (5.65), $B_{n, 1}=-H_{n}$.

Proof. This follows directly from

$$
B_{n, 1}=\sum_{j=1}^{n} \frac{(-1)^{j}}{j}\left(\begin{array}{l}
n \\
j
\end{array}\right)=b_{n}-n
$$

and the result of Lemma 5.3.

Lemma 5.5. For $2 \leq k \leq n$ and with the notation in (5.65), $B_{n, k}=-\frac{1}{k-1}$.

Proof. Observe that

$$
(k-1) B_{n, k}=\sum_{j=1}^{n}(-1)^{j}\left(\begin{array}{l}
n \\
j
\end{array}\right)\left(\begin{array}{c}
k+j-2 \\
j
\end{array}\right) .
$$

Replace $k$ by $r+2$ and observe that the result to be proven is equivalent to the identity

$$
\sum_{j=0}^{n}(-1)^{j}\left(\begin{array}{c}
n \\
j
\end{array}\right)\left(\begin{array}{c}
r+j \\
j
\end{array}\right)=0, \quad \text { for } 0 \leq r \leq n-1 \text { and } n \geq 1 .
$$

Define

$$
f(x)=\sum_{j=0}^{n}\left(\begin{array}{c}
n \\
j
\end{array}\right)\left(\begin{array}{c}
r+j \\
j
\end{array}\right) x^{j}
$$

Then (5.74) is the same as $f(-1)=0$. This is established next. The expression

$$
f(x)={ }_{2} F_{1}\left(\begin{array}{rr}
r+1 & -n \\
1 & -x
\end{array}\right)
$$

is checked directly by writing

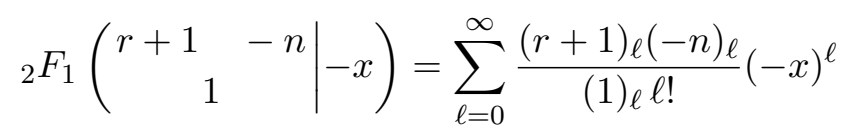

and simplifying the series using

$$
(r+1)_{\ell}=\frac{(r+\ell) !}{r !},(1)_{\ell}=\ell ! \quad \text { and }(-n)_{\ell}= \begin{cases}0 & \text { if } \ell>n \\ (-1)^{\ell} \frac{n !}{(n-\ell) !} & \text { if } \ell \leq n .\end{cases}
$$


The value

$$
f(-1)={ }_{2} F_{1}\left(\begin{array}{rr|r}
r+1 & -n \mid 1 \\
1 & \mid
\end{array}\right)
$$

is seen to vanish using Gauss' evaluation (see entry 9.122.1 in [GrRy15] and also Theorem 2.2.2 in [And99])

$$
{ }_{2} F_{1}\left(\begin{array}{cc|c}
a & b & 1 \\
c & 1
\end{array}\right)=\frac{\Gamma(c) \Gamma(c-a-b)}{\Gamma(c-a) \Gamma(c-b)} .
$$

The proof of Lemma 5.5 is complete.

The formula (5.66) and the values of $B_{n, k}$ now produce

$$
\begin{aligned}
\int_{0}^{\infty}\left[\frac{L_{n}(x)-L_{n}(0)}{x}\right]^{2} e^{-x} d x & =\sum_{k=1}^{n} \frac{(-1)^{k}}{k}\left(\begin{array}{l}
n \\
k
\end{array}\right) B_{n, k} \\
& =-n B_{n, 1}+\sum_{k=2}^{n} \frac{(-1)^{k}}{k}\left(\begin{array}{l}
n \\
k
\end{array}\right) B_{n, k} \\
& =n H_{n}-\sum_{k=2}^{n} \frac{(-1)^{k}}{k(k-1)}\left(\begin{array}{l}
n \\
k
\end{array}\right) \\
& =n H_{n}-\left(\alpha_{n}-b_{n}\right) \\
& =2 n-H_{n},
\end{aligned}
$$

as claimed.

The identity (5.74) may also be obtained from two representations of the Jacobi polynomial

$$
\begin{aligned}
P_{n}^{(\alpha, \beta)}(x) & =\frac{(-1)^{n}}{2^{n} n !}(1-x)^{-\alpha}(1+x)^{-\beta} \frac{d^{n}}{d x^{n}}\left((1-x)^{n+\alpha}(1+x)^{n+\beta}\right) \\
& =\sum_{j}\left(\begin{array}{c}
n+\alpha+\beta+j \\
j
\end{array}\right)\left(\begin{array}{c}
n+\alpha \\
n-j
\end{array}\right)\left(\frac{x-1}{2}\right)^{j} .
\end{aligned}
$$

Choose $\alpha=0, \beta=r-n$ and $x=-1$. Then (5.74) is recovered from $P_{n}^{(0, n-r)}(-1)=0$.

\section{B. The analysis for $\alpha \in \mathbb{N}$ with $\alpha \neq 1$.}

The integral

$$
W_{n}(\alpha)=\int_{0}^{\infty}\left[\frac{L_{n}^{\alpha}(x)-L_{n}^{\alpha}(0)}{x}\right]^{2} e^{-x} x^{a} d x
$$

is now discussed under the assumption that $\alpha$ is a positive integer, with the restriction $\alpha \neq 1$, extending the results for $\alpha=0$ given above. The case $\alpha=1$ is discussed in the next subsection.

Lemma 5.6. The integral $W_{n}(\alpha)$ is given by the double sum

$$
W_{n}(\alpha)=\frac{(n+\alpha) !^{2}}{n !^{2}} \sum_{j=1}^{n} \frac{(-1)^{j}}{(\alpha+j) !}\left(\begin{array}{l}
n \\
j
\end{array}\right) S_{j}(n)
$$

where

$$
S_{j}(n)=\sum_{k=1}^{n}(-1)^{k}\left(\begin{array}{l}
n \\
k
\end{array}\right) \frac{(\alpha+j+k-2) !}{(\alpha+k) !}
$$


Proof. Simply use the expression

$$
L_{n}^{\alpha}(x)=\sum_{k=0}^{n} \frac{\Gamma(\alpha+n+1)(-x)^{k}}{\Gamma(\alpha+k+1) k !(n-k) !}
$$

in (5.99), expand the square and compute the resulting integrals.

The sums $S_{j}(n)$ are now evaluated.

Lemma 5.7. The sum $S_{1}(\alpha)$ is given by

$$
S_{1}(\alpha)=\sum_{k=1}^{n} \frac{(-1)^{k}}{\alpha+k}\left(\begin{array}{l}
n \\
k
\end{array}\right)=\frac{n !(\alpha-1) !}{(\alpha+n) !}-\frac{1}{\alpha} .
$$

Proof. Integrate the expansion

$$
x^{\alpha-1}(1-x)^{n}=\sum_{k=0}^{n}(-1)^{k}\left(\begin{array}{l}
n \\
k
\end{array}\right) x^{\alpha+k-1}
$$

from $x=0$ to 1 and use the special value of the beta function

$$
\int_{0}^{1} x^{\alpha-1}(1-x)^{n} d x=B(\alpha, n+1)=\frac{(\alpha-1) ! n !}{(\alpha+n+1) !} .
$$

Now write down the term corresponding to $k=0$.

Lemma 5.8. For $j \geq 2$, the sum $S_{j}(\alpha)$ is given by

$$
S_{j}(\alpha)=\sum_{k=1}^{n}(-1)^{k}\left(\begin{array}{l}
n \\
k
\end{array}\right) \frac{(\alpha+j+k-2) !}{(\alpha+k) !}=-\frac{(\alpha+j-2) !}{\alpha !} .
$$

Proof. Observe that

$$
\frac{(\alpha+j+k-2) !}{(\alpha+k) !}=\prod_{t=1}^{j-1}(\alpha+t+k)
$$

is a polynomial in $k$ of degree $j-2<n$. Differentiating the expansion of $(1-x)^{n}$ and evaluating at $x=1$ shows that

$$
\sum_{k=0}^{n}(-1)^{k}\left(\begin{array}{l}
n \\
k
\end{array}\right) k^{\ell}=0, \quad \text { for } 0 \leq \ell \leq n-1 .
$$

Expanding (5.88) and treating the constant term separately gives the result.

The value of $W_{n}(\alpha)$ in Lemma 5.6 is now written as

$$
W_{n}(\alpha)=-\frac{(n+\alpha) !^{2}}{n !^{2}} \frac{n}{(\alpha+1) !}\left[\frac{n !(\alpha-1) !}{(\alpha+n) !}-\frac{1}{\alpha}\right]-\frac{(n+\alpha) !^{2}}{n !^{2}} \sum_{j=2}^{n} \frac{(-1)^{j}}{(\alpha+j)(\alpha+j-1) \alpha !}\left(\begin{array}{l}
n \\
j
\end{array}\right)
$$

The next step is to evaluate the sum appearing in (5.90).

Lemma 5.9. The identity

$$
\sum_{j=0}^{n} \frac{(-1)^{j}}{(\alpha+j)(\alpha+j-1) \alpha !}\left(\begin{array}{l}
n \\
j
\end{array}\right)=\frac{(n+1) !}{(n+\alpha) ! \alpha(\alpha-1)},
$$

holds. 
Proof. Write the stated identity in the form $\sum_{j=0}^{\infty} F(n, j)=1$ with

$$
F(n, j)=\frac{(-1)^{j}(n+\alpha) !}{(\alpha+j)(\alpha+j-1)(\alpha-2) !(n+1) !}\left(\begin{array}{c}
n \\
j
\end{array}\right) .
$$

The automatic methods of the WZ-theory [PWZ96] yields the companion function

$$
G(n, j)=\frac{(-1)^{j-1}(n+\alpha) !}{(\alpha+j-1)(\alpha-2) !(n+2) !}\left(\begin{array}{c}
n \\
j-1
\end{array}\right)
$$

and the identity

$$
F(n+1, j)-F(n, j)=G(n, j+1)-G(n, j) .
$$

Summing over $j$ shows that the sum of $F(n, j)$ is independent of $n$. Direct evaluation at $n=0$ shows this sum is 1 .

It follows that the sum in (5.90) is given by

$$
\sum_{j=2}^{n} \frac{(-1)^{j}}{(\alpha+j)(\alpha+j-1) \alpha !}=\frac{(n+1) !}{(\alpha+n) ! \alpha(\alpha-1)}-\frac{1}{\alpha ! \alpha(\alpha-1)}+\frac{n}{\alpha ! \alpha(\alpha+1)} .
$$

An expression for the integral associated to the Laguerre polynomials is presented next,

Theorem 5.10. For $\alpha \geq 2$ an integer, the integral

$$
W_{n}(\alpha)=\int_{0}^{\infty}\left[\frac{L_{n}^{\alpha}(x)-L_{n}^{\alpha}(0)}{x}\right]^{2} e^{-x} x^{a} d x
$$

is given by

$$
W_{n}(\alpha)=(\alpha-2) !\left(\begin{array}{c}
\alpha+n \\
\alpha
\end{array}\right)\left[\left(\begin{array}{c}
\alpha+n \\
\alpha
\end{array}\right)-\frac{2 n \alpha+\alpha+1}{\alpha+1}\right]
$$

Proof. Replace the values for the sums $S_{j}(n)$ computed before and simplify in the expression in Lemma 5.6.

Note 5.11. Let $(u)_{r}=u(u+1) \cdots(u+r-1)$ be the Pochhammer symbol. The explicit expression in (5.96) shows that the function $V_{\alpha}(n)$ defined by

$$
\int_{0}^{\infty}\left[\frac{L_{n}^{\alpha}(x)-L_{n}^{\alpha}(0)}{x}\right]^{2} e^{-x} x^{a} d x=V_{\alpha}(n)(n)_{\alpha+1},
$$

is a polynomial in $n$, given by

$$
V_{\alpha}(n)=\frac{1}{n \alpha(\alpha-1)}\left[\left(\begin{array}{c}
\alpha+n \\
\alpha
\end{array}\right)-\frac{2 n \alpha}{\alpha+1}-1\right] .
$$

\section{C. The analysis for $\alpha=1$.}

The integral

$$
W_{n}(\alpha)=\int_{0}^{\infty}\left[\frac{L_{n}^{\alpha}(x)-L_{n}^{\alpha}(0)}{x}\right]^{2} e^{-x} x^{\alpha} d x
$$

is now evaluated when $\alpha=1$. 
Start with the identity

$$
W_{n}(1)=\sum_{k=1}^{n} \frac{(n+1) !^{2}(-1)^{k}}{(k+1) ! k !(n-k) !} S_{k}(n)
$$

with

$$
S_{k}(n)=\sum_{j=1}^{n} \frac{(-1)^{j}(k+j-1) !}{(j+1) ! j !(n-j) !}
$$

obtained by direct expansion of the integrand in $W_{n}(1)$.

Lemma 5.12. For $1 \leq k \leq n$, the sum $S_{k}(n)$ is given by

$$
S_{k}(n)=-\frac{1}{n !} \begin{cases}\frac{n}{n+1} & \text { if } k=1 \\ (k-1) ! & \text { if } k>1\end{cases}
$$

Proof. The case $k=1$ is equivalent to the identity

$$
\sum_{j=0}^{n} \frac{(-1)^{j}}{j+1}\left(\begin{array}{l}
n \\
j
\end{array}\right)=\frac{1}{n+1}
$$

which follows by direct integration over $[0,1]$ of the expansion of $(1-x)^{n}$. The case $k \geq 2$ is equivalent to

$$
\sum_{j=0}^{n}(-1)^{j}\left(\begin{array}{l}
n \\
j
\end{array}\right) \times \frac{(k+j-1) !}{(j+1) !}=0 .
$$

This follows from the fact that $(k+j-1) ! /(j+1)$ ! is a polynomial in $j$ of degree $k-2<n$ and the classical value stated in (5.89).

Replacing these values in (5.100) gives, after some simplification,

$$
W_{n}(1)=\frac{1}{2} n^{2}(n+1)-(n+1)^{2} \sum_{k=2}^{n} \frac{(-1)^{k}}{k(k+1)}\left(\begin{array}{l}
n \\
k
\end{array}\right) .
$$

Theorem 5.13. The integral $W_{n}(1)$ is given by

$$
W_{n}(1)=-\frac{1}{2}(3 n+2)(n+1)+(n+1)^{2} H_{n+1},
$$

where $H_{n+1}$ is the harmonic number.

Proof. This follows from (5.105) and the value

$$
\sum_{k=1}^{n} \frac{(-1)^{k}}{k(k+1)}\left(\begin{array}{l}
n \\
k
\end{array}\right)=1-H_{n+1}
$$

This evaluation follows from

$$
\sum_{k=1}^{n}(-1)^{k}\left(\begin{array}{l}
n \\
k
\end{array}\right) \frac{x^{k+1}}{k(k+1)}=\int_{0}^{x} \int_{0}^{t} \frac{\left(1-x_{1}\right)^{n}-1}{x_{1}} d x_{1} d t
$$

by exchanging the order of integration and using the evaluation of (5.69). 


\section{The Gegenbauer case}

The Gegenbauer polynomial $C_{n}^{(a)}(x)$ is defined by the explicit expression

$$
C_{n}^{(a)}(x)=\sum_{k=0}^{\left\lfloor\frac{n}{2}\right\rfloor}(-1)^{k} \frac{\Gamma(n-k+a)}{\Gamma(a) k !(n-2 k) !}(2 x)^{n-2 k},
$$

or by the recurrence

$$
C_{n}^{(a)}(x)=\frac{1}{n}\left[2 x(n+a-1) C_{n-1}^{(a)}(x)-(n+2 a-2) C_{n-2}^{(a)}(x)\right],
$$

with initial conditions $C_{0}^{(a)}(x)=1$ and $C_{1}^{(a)}(x)=2 a x$. The Gegenbauer polynomials are orthogonal in $[-1,1]$ with respect to the weight $\left(1-x^{2}\right)^{a-1 / 2}$ and are normalized by

$$
\int_{-1}^{1}\left[C_{n}^{a}(x)\right]^{2}\left(1-x^{2}\right)^{a-1 / 2} d x=\frac{\pi \Gamma(n+2 a)}{2^{2 a-1} n !(n+a) \Gamma^{2}(a)} .
$$

The integral discussed in this section is given by

$$
\gamma_{n}(a)=\int_{-1}^{1}\left[\frac{C_{n}^{a}(x)-C_{n}^{a}(0)}{x}\right]^{2}\left(1-x^{2}\right)^{a-1 / 2} d x .
$$

Proceeding as in the previous sections one may obtain a recurrence for these integrals.

Lemma 6.1. The integrals $\gamma_{n}(a)$ satisfy the recurrence

$$
\gamma_{n}(a)=\frac{\pi(n+a-1) \Gamma(n-1+2 a)}{2^{2 a-3} n n ! \Gamma^{2}(a)}+\frac{(n+2 a-2)^{2}}{n^{2}} \gamma_{n-2}(a)
$$

with initial conditions

$$
\gamma_{0}(a)=0 \quad \text { and } \quad \gamma_{1}(a)=\frac{4 a \sqrt{\pi} \Gamma\left(a+\frac{1}{2}\right)}{\Gamma(a)}
$$

From the recurrence (6.113), the value $\gamma_{2 m+1}(a)$ is easy to realize. Let $n=2 m+1$ and write the recurrence as

$$
\gamma_{2 m+1}(a)=\frac{\pi(2 m+a) \Gamma(2 m+2 a)}{2^{2 a-3}(2 m+1)(2 m+1) ! \Gamma^{2}(a)}+\frac{(2 m+2 a-1)^{2}}{(2 m+1)^{2}} \gamma_{2 m-1}(a) .
$$

Proposition 6.2. For $m \in \mathbb{N}$ odd, the integral $\gamma_{m}(a)$ is given by

$$
\gamma_{m}(a)=\frac{\pi \Gamma(2 a+m)}{2^{2 a-2} m ! \Gamma^{2}(a)}
$$

Proof. Introduce the new variable $u_{m}$ by

$$
u_{m}(a)=\left(\frac{\pi \Gamma(2 a+2 m+1)}{2^{2 a-2}(2 m+1) ! \Gamma^{2}(a)}\right)^{-1} \gamma_{2 m+1}(a)
$$

and convert (6.115) into

$$
(a+m)(2 m+1) u_{m}(a)=2 m+a+m(2 m+2 a-1) u_{m-1}(a) .
$$

An inductive argument now shows that $u_{m}(a) \equiv 1$, proving the assertion. 
The next results present the evaluation of $\gamma_{n}(a)$ for $n$ even. The recurrence (6.113) is now used to produce data, motivating the scaling

$$
X_{n}(a)=\frac{\Gamma(a)(2 n) !}{4 \sqrt{\pi} \Gamma\left(a+\frac{1}{2}\right)(a)_{n}} \gamma_{2 n}(a)
$$

where $(a)_{n}$ is the Pochhammer symbol. Then (6.113) yields:

Proposition 6.3. The function $X_{n}(a)$ satisfies the recurrence

$$
X_{n}(a)=\frac{2}{n}(a+n-1)(2 n-1) X_{n-1}(a)+\frac{(2 n+a-1)}{n} 2^{2 n-2}\left(a+\frac{1}{2}\right)_{n-1},
$$

with $X_{0}(a)=0$. Therefore $X_{n}(a)$ is a polynomial in a of degree $n$.

Corollary 6.4. The coefficients of $X_{n}(a)$ are positive.

A second recurrence for $X_{n}(a)$ is obtained by eliminating the Pochhammer symbol in the result of Proposition 6.3.

Proposition 6.5. The polynomials $X_{n}(a)$ satisfy the recurrence

$$
\begin{aligned}
n(2 n+a-3) X_{n}(a)= & 2(2 n+a-2)\left(4 n^{2}+4 a n-8 n-3 a+3\right) X_{n-1}(a) \\
& -4(a+n-2)(2 n-3)(2 a+2 n-3)(2 n+a-1) X_{n-2}(a),
\end{aligned}
$$

with initial conditions $X_{0}(a)=0$ and $X_{1}(a)=a+1$.

The next result gives a zero for the polynomial $X_{n}(a)$. From the proof one obtains alternative expressions for this polynomial. In turn, these produce a simpler proof of the theorem below. The original proof presented here also has pedagogical interest.

Theorem 6.6. The polynomial $X_{n}$ vanishes at $a=-n$.

Proof. Introduce the function $f_{n}(a)=2(a+n-1)(2 n-1) / n$ and divide the recurrence for $X_{n}$ by

$$
\prod_{j=1}^{n} f_{j}(a)=\frac{(n+a-1) !}{(a-1) !}\left(\begin{array}{c}
2 n \\
n
\end{array}\right)
$$

with $a !=\Gamma(a+1)$ for non-integer $a$. This yields

$$
A_{n}(a)-A_{n-1}(a)=\frac{(2 n+a-1)}{n a} \frac{\left(\begin{array}{c}
2 n+2 a-2 \\
n+a-1
\end{array}\right)}{\left(\begin{array}{c}
2 n \\
n
\end{array}\right)\left(\begin{array}{c}
2 a \\
a
\end{array}\right)},
$$

with $A_{n}(a)$ being the quotient of $X_{n}(a)$ by the product in (6.120). Summing over $n$ gives

$$
X_{n}(a)=\frac{(n+a-1) !\left(\begin{array}{c}
2 n \\
n
\end{array}\right)}{a !\left(\begin{array}{c}
2 a \\
a
\end{array}\right)} \sum_{k=1}^{n} \frac{(2 k+a-1)}{k\left(\begin{array}{c}
2 k \\
k
\end{array}\right)}\left(\begin{array}{c}
2 k+2 a-2 \\
k+a-1
\end{array}\right) .
$$

This can be written as

$$
X_{n}(a)=(n-1) !\left(\begin{array}{c}
a+n-1 \\
n-1
\end{array}\right)\left(\begin{array}{c}
2 n \\
n
\end{array}\right) \sum_{k=1}^{n} \frac{(2 k+a-1) a^{2}}{2 k^{2}(2 k-1)} \frac{\left(\begin{array}{c}
2 a+2 k-2 \\
2 k-2
\end{array}\right)}{\left(\begin{array}{c}
a-1+k \\
k
\end{array}\right)^{2}}
$$


and using $\left(\begin{array}{c}-x+y \\ y\end{array}\right)=(-1)^{y}\left(\begin{array}{c}x-1 \\ y\end{array}\right)$ gives

$$
X_{n}(a)=\frac{(-1)^{n-1}}{2}(n-1) !\left(\begin{array}{c}
-a-1 \\
n-1
\end{array}\right)\left(\begin{array}{c}
2 n \\
n
\end{array}\right) a^{2} \sum_{k=1}^{n} \frac{(2 k+a-1)}{k^{2}(2 k-1)} \frac{\left(\begin{array}{c}
-2 a-1 \\
2 k-2
\end{array}\right)}{\left(\begin{array}{c}
-a \\
k
\end{array}\right)^{2}} .
$$

Using this form of $X_{n}(a)$ it follows that $X_{n}(-n)=0$ is equivalent to the identity

$$
\sum_{k=1}^{n} \frac{(2 k-1-n)}{k^{2}(2 k-1)} \frac{\left(\begin{array}{l}
2 n-1 \\
2 k-2
\end{array}\right)}{\left(\begin{array}{l}
n \\
k
\end{array}\right)^{2}}=0
$$

whose proof is based on the automatic methods developed by H. Wilf and D. Zeilberger [PWZ96]. To this end, denote the summand in (6.124) by

$$
F(n, k)=\frac{(2 k-1-n)}{k^{2}(2 k-1)} \frac{\left(\begin{array}{l}
2 n-1 \\
2 k-2
\end{array}\right)}{\left(\begin{array}{l}
n \\
k
\end{array}\right)^{2}}
$$

and then using the WZ-methodology gives the companion function

$$
G(n, k)=\frac{(k-n-1)\left(\begin{array}{c}
2 n-1 \\
2 k-2
\end{array}\right)}{k^{2}\left(\begin{array}{l}
n \\
k
\end{array}\right)^{2}}
$$

to the effect that

$$
F(n, k)=G(n, k+1)-G(n, k) .
$$

Now sum from $k=1$ to $n$ and use the values $G(n, n+1)=G(n, 1)=-1 / n$ to obtain the result.

Note 6.7. The alternative expression for $X_{n}(a)$ given below follow from elementary manipulations of those appearing in the previous proof. The formula is written in terms of factorials (with the usual interpretation

$b !=\Gamma(b+1)$ for $b \notin \mathbb{N})$. The formula is

$$
X_{n}(a)=\frac{1}{8}\left(\begin{array}{c}
2 n \\
n
\end{array}\right) \frac{(a+n-1) !}{\left(a-\frac{1}{2}\right) !} \sum_{k=1}^{n} \frac{(2 k+a-1)}{(2 k-1)} \frac{1}{\left(\begin{array}{c}
2 k-2 \\
k-1
\end{array}\right)} \frac{\left(a+k-\frac{3}{2}\right) !}{(a+k-1) !} 2^{2 k}
$$

A simpler expression for $X_{n}(a)$ is given next.

Theorem 6.8. For $n \geq 1$, the polynomial $X_{n}$ is given by

$$
\begin{aligned}
X_{n}(a) & =2^{2 n-1}\left(a+\frac{1}{2}\right)_{n}-\left(\begin{array}{c}
2 n-1 \\
n-1
\end{array}\right)(a)_{n} \\
& =2^{n-1} \prod_{k=0}^{n-1}(2 a+2 k+1)-\left(\begin{array}{c}
2 n-1 \\
n-1
\end{array}\right) \prod_{k=0}^{n-1}(a+k) .
\end{aligned}
$$

Proof. The formula (6.128) is now written in term of Pochhammer symbols as

$$
X_{n}(a)=\frac{1}{8}\left(\begin{array}{c}
2 n \\
n
\end{array}\right) \sum_{k=1}^{n} \frac{2^{2 k}}{(2 k-1)\left(\begin{array}{c}
2 k-2 \\
k-1
\end{array}\right)}\left[(2 k+a-1)\left(a+\frac{1}{2}\right)_{k-1}(a+k)_{n-k}\right] .
$$

A symbolic evaluation of (6.130), using Mathematica, leads to (6.129). To prove this assertion, simply verify that the right-hand side satisfies (6.3) for $n \geq 1$ and that both sides give $a+1$ at $n=1$. 
Some elementary properties of the polynomial $X_{n}(a)$ can be obtained from (6.129). The first class of results deal with the coefficients.

Property 6.9. The leading coefficient of $X_{n}(a)$ is $2^{2 n-1}-\frac{1}{2}\left(\begin{array}{c}2 n \\ n\end{array}\right)$.

Property 6.10. The constant term in $X_{n}(a)$ is $\frac{(2 n) !}{2 n !}$.

Property 6.11. The expansion

$$
(x)_{n}=\sum_{j=0}^{n}(-1)^{n-j} s(n, j) x^{j},
$$

where $s(n, j)$ are the Stirling numbers of the first kind is now replaced in (6.130) to produce an expression for the coefficients in

$$
X_{n}(a)=\sum_{r=0}^{n} \rho_{n, r} a^{r}
$$

in the form

$$
\rho_{n, r}=(-1)^{n} 2^{n+1-r}\left[\sum_{j=r}^{n}(-1)^{j} 2^{n-j}\left(\begin{array}{l}
j \\
r
\end{array}\right) s(n, j)-(-1)^{r} 2^{n}\left(\begin{array}{c}
2 n-1 \\
n-1
\end{array}\right) s(n, r)\right]
$$

Therefore the coefficients $\rho_{n, r}$ is an integer.

Corollary 6.12. The coefficients $\rho_{n, r}$ are positive integers.

Proof. This follows from (6.133) and Corollary 6.4.

The second type of results relate to the values of $X_{n}$. The first statement is another proof of Theorem 6.6.

Property 6.13. The polynomial $X_{n}(a)$ vanishes at $a=-n$.

Proof. This follows from the values

$$
\left(-n+\frac{1}{2}\right)_{n}=(-1)^{n} \frac{(2 n) !}{n ! 2^{2 n}} \text { and }(-n)_{n}=(-1)^{n} n !,
$$

and (6.129).

Property 6.14. For $n \in \mathbb{N}$ and $1-n \leq k \leq-1$, we have

$$
X_{n}(-k)=(-1)^{k} 2^{2 n-1}\left(\frac{1}{2}\right)_{k}\left(\frac{1}{2}\right)_{n-k} .
$$

and they satisfy the symmetry condition

$$
X_{n}(k-n)=(-1)^{n} X_{n}(-k), \quad \text { for } 1 \leq k \leq n-1 .
$$

Proof. This follows directly from (6.129) and the identities

$$
\left(k-n+\frac{1}{2}\right)_{n}=(-1)^{n}\left(\frac{1}{2}-k\right)_{n} \quad \text { and } \quad(k-n)_{n}=(-1)^{n}(-k)_{n} .
$$

Property 6.15. All zeros of $X_{n}(a)$ are real and negative. 
Proof. The value $X_{n}(0)$, given in Property 6.10, is positive. On the other hand, Property 6.14 shows that $X_{n}(-1)<0$. Therefore, there is a real zero of $X_{n}(a)$ in the interval $(-1,0)$. Property 6.14 shows that $X_{n}(-1)<0$. The signs of $X_{n}(j)$ alternate for $j=-1,-2, \cdots,-n+1$ giving a zero in the open interval $(j-1, j)$ as shown before for $j=0$. This accounts for $n-1$ real negative zeros. Property 6.13 shows that there is one more zero exactly at $a=-n$, for a total of $n$. Since $X_{n}(a)$ is of degree $n$, these are all of them.

The next statement looks at the polynomial $X_{n}(a)$ modulo $n$, when $n$ is prime.

Property 6.16. For q prime

$$
X_{q}(a) \equiv a^{q}-a \bmod q .
$$

Proof. From $2^{q-1} \equiv 1 \bmod q$, we obtain

$$
2^{2 q-1}\left(a+\frac{1}{2}\right)_{q} \equiv(2 a+1)(2 a+3) \cdots(2 a+2 q-1) \bmod q .
$$

Wolstelholme theorem gives

$$
\left(\begin{array}{c}
2 q-1 \\
q-1
\end{array}\right) \equiv 1 \bmod q
$$

so that

$$
X_{q}(a) \equiv(2 a+1)(2 a+3) \cdots(2 a+2 q-1)-a(a+1) \cdots(a+q-1) \bmod q .
$$

The lists $\{1,3, \cdots, 2 q-1\}$ and $\{0,2, \cdots, 2 q-2\}$ are both the same list as $\{1, \cdots, q\}$ modulo $q$. Therefore

$$
\begin{aligned}
X_{q}(a) & \equiv \prod_{j=1}^{q}(2 a+2 j-1)-\prod_{j=1}^{q}(a+j) \\
& \equiv \prod_{j=1}^{q}(2 a+j)-\prod_{j=1}^{q}(a+j) \\
& \equiv \prod_{j=1}^{q}(2 a+2 j)-\prod_{j=1}^{q}(a+j) \\
& \equiv\left(2^{q}-1\right) \prod_{j=1}^{q}(a+j) \\
& \equiv \prod_{j=1}^{q}(a+j),
\end{aligned}
$$

all congruences being taken modulo $q$. In the finite field $\mathbb{F}_{q}$, the polynomial $f(a)=a^{q}-a$ factors completely with roots $1,2, \cdots, q$. Hence

$$
a^{q}-a \equiv \prod_{k=1}^{q}(a-k) \equiv \prod_{k=1}^{q}(a+k) \bmod q .
$$

This completes the proof.

We conclude the discussion on the polynomials $X_{n}(a)$ with a question based on extensive Mathematica computations: 
Problem. Define $Z_{n}(a)=X_{n}(a) /(a+n)$. Then the family of polynomials $\left\{Z_{n}(a)\right\}_{n \geq 1}$ have the interlacing property; that is, the roots of $Z_{n}$ interlace those of $Z_{n-1}$.

Acknowledgement. The authors wish to thank C. Koutschan for providing the result in Property 6.14. The authors also wish to thank a referee for a question that motivated the results on Laguerre polynomials presented in Section 5 .

\section{References}

[And99] G.E. Andrews, R. Askey and R. Roy, Special Functions, Encyclopedia of Mathematics and its Applications (Volume 71), Cambridge University Press, New York, 1999.

[DiKo12] E. Diekema and T. Koorwinder, Generalizations of an integral for Legendre polynomials by Persson and Strang, J. Math.Anal. Appl., 388 (2012)125-135.

[GrRy15] I.S. Gradshteyn and I.M. Ryzhik, Table of Integrals, Series, and Products, Edited by D. Zwillinger and V. Moll. Academic Press, New York, 8th edition, 2015.

[PeSt03] P.E. Persson and G. Strang, Smoothing by Savitzky-Golay and Legendre filters, Mathematical systems theory of biology, communications, computations, and finance, volume 134 of IMA Vol. Math. Appl., (2003) 301-316.

[PWZ96] M. Petkovsek, H. Wilf, and D. Zeilberger, $A=B$ A. K. Peters, 1st. edition, 1996.

\section{T. Amdeberhan}

Department of Mathematics

Tulane University

New Orleans, LA 70118, USA

e-mail: tamdeber@tulane.edu

Adriana Duncan

Department of Mathematics

Tulane University

New Orleans, LA 70118, USA

and

Department of Mathematics

University of Texas at Austin

TX 78712, USA

e-mail: aduncan3@tulane.edu and aduncan@utexas.edu

\section{Victor H. Moll}

Department of Mathematics

Tulane University

New Orleans, LA 70118, USA

e-mail:vhm@tulane.edu

\section{Vaishavi Sharma}

Department of Mathematics

Tulane University

New Orleans, LA 70118, USA

e-mail:vsharma1@tulane.edu 